\title{
Resveratrol Treatment Prevents Hippocampal Neurodegeneration in a Rodent Model of Traumatic Brain Injury
}

\author{
Tugay ATALAY ${ }^{1}$, Ismail GULSEN², Nese COLCIMEN ${ }^{3}$, Hamit Hakan ALP ${ }^{4}$, Enver SOSUNCU ${ }^{5}$, Ilker ALACA ${ }^{6}$, \\ Hakan AK ${ }^{1}$, Murat Cetin RAGBETLI ${ }^{3}$
}

${ }^{1}$ Bozok University, School of Medicine, Department of Neurosurgery, Yozgat, Turkey

${ }^{2}$ Yuzuncu YII University, Department of Neurosurgery, Van, Turkey

${ }^{3}$ Yuzuncu YIl University, Department of Histology and Embryology, Van, Turkey

${ }^{4}$ Yuzuncu Yıl University, Department of Biochemistry, Van, Turkey

${ }^{5}$ Ercis State Hospital, Department of Neurosurgery, Van, Turkey

${ }^{6}$ Yuksekova State Hospital, Department of Neurosurgery, Hakkari, Turkey

\section{ABSTRACT}

AIM: Traumatic brain injury (TBI) is a complex process. Increasing evidence has demonstrated that reactive oxygen species contribute to brain injury. Resveratrol (RVT) which exhibits significant antioxidant properties, is neuroprotective against excitotoxicity, ischemia, and hypoxia. The aim of this study was to evaluate the neuroprotective effects of RVT on the hippocampus of a rat model of TBI.

MATERIAL and METHODS: Twenty eight rats were divided into four groups. A moderate degree of head trauma was induced using Feeney's falling weight technique. Group 1 (control) underwent no intervention or treatment. Head trauma was induced in Group 2 (trauma) and no drug was administered. Head trauma was induced in Group 3 and low-dose RVT (50 mg/kg per day) was injected. In Group 4, high-dose RVT (100 mg/kg per day) was used after head trauma. Brain tissues were extracted immediately after perfusion without damaging the tissues. Histopathological and biochemistry parameters were studied.

RESULTS: Brain tissue malondialdehyde (MDA) levels in the trauma group were significantly higher than those in the control, lowdose RVT-treated, and high-dose-RVT-treated groups. The superoxide dismutase (SOD) levels in the control group were significantly higher than those in the trauma, low-dose RVT-treated, and high-dose RVT-treated groups. Glutathione peroxidase (GSH-Px) levels in the control group were significantly higher than those in the trauma and low-dose RVT-treated groups. The level of oxidative deoxyribonucleic acid (DNA) damage (8-OHdG/106 dG) in the trauma group was higher than that in the control group, low-dose RVT-treated, and high-dose RVT-treated groups.

CONCLUSION: Resveratrol has a healing effect on neurons after TBI.

KEYWORDS: Resveratrol, Traumatic brain injury, Hippocampus, Malondialdehyde, Superoxide dismutase 


\section{INTRODUCTION}

$\mathrm{T}$ raumatic brain injury (TBI) occurs as the result of a complicated series of primary and secondary injuries and regenerative processes (35). Secondary injuries manifest in the hours and days after TBI due to intricate biochemical and physiological mechanisms $(15,29,30)$. Several abnormal functions including cell toxicity, inflammation and reactive oxygen species (ROS), and mitochondrial dysfunction are all implicated in the sequelae of TBI. These processes exacerbate brain trauma through edema $(15,25,28)$. Proper and timely intervention can limit the secondary injuries to the brain and reduce the overall mortality rates in patients $(15,41)$.

Widespread evidence has shown that ROS negatively impacts TBI (13). Enzymes that produce catalase (CAT) and glutathione peroxidase (GSH-Px) have been shown to mitigate the effects of free oxygen radicals (10).

Resveratrol (RVT), a naturally occurring compound of the stilbene class, is a polyphenolic molecule, which is found in grapes, berries, red wine, chocolate, and root vegetables. RVT gained popularity after publication of a 1993 paper describing the prevention of low density lipoprotein oxidation by RVT, which suggested that RVT could be used to treat atherosclerosis (14). RVT is a potent anti-oxidant and neuroprotective agent shown to protect neural tissue from the damaging effects of hypoxia ischemia, and cell toxicity in both cell culture and animal models $(4,9,20,44)$. Some studies have found that TBI in both adult humans and animals is amenable to treatment with RVT (5).

In this study, we examined the efficacy of RVT using biochemical and histopathological parameters on the hippocampus of experimental rodent model of TBI.

\section{MATERIAL and METHODS}

\section{Animals and Ethical Standards}

Female Wistar albino rats (250 to 300 grams) were housed and raised at the Animal Care Center, Yuzuncu Yıl University, Van, Turkey. Animals were kept in metabolic cages at $25^{\circ} \mathrm{C}$ with a 12-on/12-off light/dark cycle. All rats were fed standard chow and water. The Yüzüncü Yıl University ethics committee reviewed and approved all animal procedures (YÜHADYEK; date: 30.01 .2014 , number: 2014/02).

\section{Chemicals}

RVT (Sigma Chemicals, St. Louis, MO, USA) was suspended in saline. 50 and $100 \mathrm{mg} / \mathrm{kg}$, doses of RVT were prepared for the RVT groups and equal amounts of physiological saline were used for treatment immediately after trauma (RVT, Santa Clara, CA, USA).

\section{Experimental Protocol}

The experiment included 28 animals that were divided equally between 4 groups. All animals were weighed at baseline and at the end of the experiment. Anesthesia consisted of intra-peritoneal $80 \mathrm{mg} / \mathrm{kg}$ ketamine and $10 \mathrm{mg} / \mathrm{kg}$ xylazine administration. An adequate level of trauma to the cranium was done using Feeney's falling weight (FFW) method (12). Briefly, a $9 \mathrm{~g}$ brass cylinder was dropped onto the rat cranium from a height of $0.05 \mathrm{~m}$ using a vertical glass cylinder to control the fall of the weight. The falling weight distance was determined using the formula:

$$
\mathrm{E}=m g \times h,
$$

where $\mathrm{E}=$ energy, $\mathrm{mg}=$ mass $\mathrm{x}$ gravity or weight, and $\mathrm{h}=$ height . This system allowed for precise and reproducible trauma in all experimental animals. After anesthetization, rats were leveled on a hard surface and subjected to a dermal mid-line incision. A dental drill at the right side of the midline was used to make a $10-\times 15-\mathrm{mm}$ area. A $1-\times 1-\mathrm{cm}$ disc was placed into the cranial area. The animals were then re-positioned on a foam pad and TBI was induced using Feeney's device.

Rats were categorized in to four groups:

Group 1: Rats did not undergo TBI or any other intervention.

Group 2: Rats underwent TBI using FFW method but were not treated with any drug.

Group 3: Rats underwent TBI using FFW method and were treated with low-dose RVT (50 mg/kg per day) by intraperitoneal (IP) injection for 7 days.

Group 4: Rats underwent TBI and were treated with high-dose RVT (100 mg/kg per day). Following TBI or sham TBI, all animals were returned to standard care for 8 days. No rats died during the study. Following the procedure, the rats in each group were anesthetized as described above and underwent thoracotomy. Intra-cardiac perfusion was conducted for 5 minutes using physiological serum. Immediately after perfusion, brain tissues were carefully extracted to avoid additional damage to the tissue. These tissues were divided into two parts and included "area of trauma" and both "frontoparietal zones". Half of the tissue was fixed in $10 \%$ formalin for histopathological and microscopical analysis, while the others were collected and stored for a later use.

\section{Biochemical Evaluations}

Malondialdehyde (MDA): We used high-performance liquid chromatography (HPLC) to measure serum MDA levels according to the method as previously described (23). Fluorescence was used to detect the MDA-TBA complex (ex:527 nm and em:551 nm) with an Agilent 1260 HPLC-FLD system (Agilent Technologies, Waldbronn, Germany). MDATBA $(\mu \mathrm{M})$ was determined using 1,1,3,3-tetraethoxypropane as a standard.

Isolation and hydrolysis of Deoxyribonucleic acid (DNA): DNA extraction from leukocytes was performed as previously described with slight modifications (2). Briefly, nuclear extracts were acquired from $2 \mathrm{~mL}$ aliquots of whole blood. DNA extracts were prepared using prior methods (22). The DNA was reconstituted in an eluent before the procedure followed by detection and quantification of 8-Hydroxydeoxyguanosine (8-OHdG) and deoxyguanosine (dG) levels using HPLC. The $\mathrm{dG}$ levels were measured by absorbance (at $245 \mathrm{~nm}$ ), and 8-OHdG was measured using electrochemical methods $(600 \mathrm{mV})$. Oxidative DNA damage was reported as $8-\mathrm{OHdG}$ molecules $/ 10^{6} \mathrm{dG}$ (39). 
Glutathione peroxidase (GSH-Px) analysis: 1-chloro2,4-dinitrobenzene and GSH were used to measure GSH S-transferase activity. GSH activity was measured spectrophotometrically at $340 \mathrm{~nm}$, using oxidized GSH and NADPH.

Superoxide dismutase (SOD) analysis: SOD was quantified using methods as described (36). Xanthine-xanthine oxidase yields superoxide radicals that when combined with nitroblue tetrazolium (NBT) generate a colored formazan. SOD activity was quantified as the inhibition of the reaction with NBT. SOD activity is expressed in $\mathrm{IU} / \mathrm{mL}$.

\section{Histopathological Evaluation}

Animals were sacrificed after exsanguination. The brain tissues were harvested during the craniectomy, paraffin-embedded tissues were prepared, and sections $(5-\mathrm{mm})$ were made. Sections were generated for each animal, and subjected to hematoxylin and eosin (H\&E) staining and the hippocampus was examined using a $63 \mathrm{X}$ oil objective (NA = 1.25) (Figure $1 \mathrm{~A}, \mathrm{~B})$. The dissect-Cavalieri principle was used to calculate the change hippocampal neuron amount. Total tissue volume ratios were calculated using the Shetereo (ver. 1.5) software $(16,19)$. The formula below was used to count the pyramidal neurons:

$$
N(T P N N)=\bar{Q}^{-} \times \bar{Q} P \times k \times(a / p)
$$

a(frame)2

where $\mathrm{N}=$ pyramidal neuron number, $\overline{\mathrm{Q}}^{-}=$pyramidal neurons counted in each field, QP = number of points within the hippocampal area, $k=$ section sampling fraction (1/100), and $a / p=$ area of each point on the point-counting grid of the counting frame. The data were analyzed with the KruskalWallis test. The coefficient of error was also estimated (16).

\section{Statistical Analysis}

Statistics for the variables were represented as median, mean, standard deviation, and range. Variation between multiple groups was compared using the Kruskal-Wallis test. Dunn's multiple-comparison test was used to detect specific differences among groups. All analyses were conducted using SPSS software (ver. 13; SPSS Inc., Chicago, IL, USA). A $p$-value of less than 0.05 was considered statistically significant.

\section{RESULTS}

\section{Histopathological Findings}

The neuron density in CA1 region of the four different treatment groups was 155.70 (range, 142.25-248.22), 95.45 (range, 64.42-105.20), 107.19 (range, 67.38-121.81), and 112.52 (range, 61.38-182.41), respectively. The neuron density in CA23 region was 90.68 (range, 84.97-95.70), 59.51 (26.04-92.05), 68.25 (41.29-175.64), and 78.07 (30.26-64.62), respectively. The neuron density in the contralateral hippocampal CA4 region was 45.25 (40.20-57.40), 25.57 (10.90-30.40), 29.69 (28.60-34.30), and 38.15 (30.50-50.20), respectively.

There were significant differences in the neuron density among the groups $(p<0.05)$. The neuron densities in the contralateral hippocampal regions (CA1 and CA4) were lower in the trauma and RVT-treated groups relative to the controls $(p<0.001)$. Neuron densities in CA1, CA2-3, and CA4 were higher in the high-dose RVT-treated animals relative to the trauma animals


Figure 1: A) CA2-3 hippocampus section stained by H\&E at 63x magnification (Group of RVT 50). B) CA2-3 hippocampus section stained by H\&E at 63x magnification (Group of RVT 100). 
$(p<0.001)$. Although the neuron densities in CA1, CA2-3, and CA4 were higher in the low-dose RVT-treated group compared to the trauma group, there was no significant difference (Figure 2).

\section{Biochemical Findings}

Brain tissue MDA measurements in the animals with trauma were significantly elevated relative to the control, low-dose RVT-treated, and high-dose-RVT-treated groups (5.18, 3.28, 3.45 , and 4.20, respectively; $\mathrm{p}=0.029$ ). However, MDA was not significantly different between the RVT-treated animals (low and high dose) and the controls $(p=0.49)$ (Figure 3$)$. In addition, the SOD activity was increased in the controls relative to the trauma, low-dose RVT-treated, and high-dose RVT-treated groups $(10.75,5.32,5.43$, and 6.10, respectively; $p=0.002$ ). However, SOD activity did not differ between the others groups $(p=0.893)$ (Figure 4$)$.

GSH-Px activity in the control animals was significantly elevated relative to the trauma and low-dose RVT-treated groups $(12.93,6.92$, and 6.55 , respectively; $p=0.006$ ).
Conversely, there was no change in GSH-Px activity between the control animals and animals treated with high dose RVT $(p=0.643)$. GSH-Px levels in the high-dose RVT-treated animals were significantly elevated relative to the trauma animals (11.12 and 6.92, respectively; $p=0.079$ ) (Figure 5).

Oxidative DNA damage (8-OHdG/106 dG) was elevated in the trauma group relative to the control-treated animals and the low-dose RVT-treated, and high-dose RVT-treated groups $(2.21,1.54,1.61$, and 1.76 , respectively), however this difference was not significant ( $p>0.05$ ) (Figure 6).

\section{DISCUSSION}

In this report, we demonstrated that RVT injections significantly reduced histological and biochemical signs of neuronal damage in a rodent model of TBI.

Impacts to the brain incur injury in two stages. Primary injury is the damage brought on by the impact itself and occurs immediately. Secondary injuries result from ischemia and hypotension resulting from the pathophysiological processes

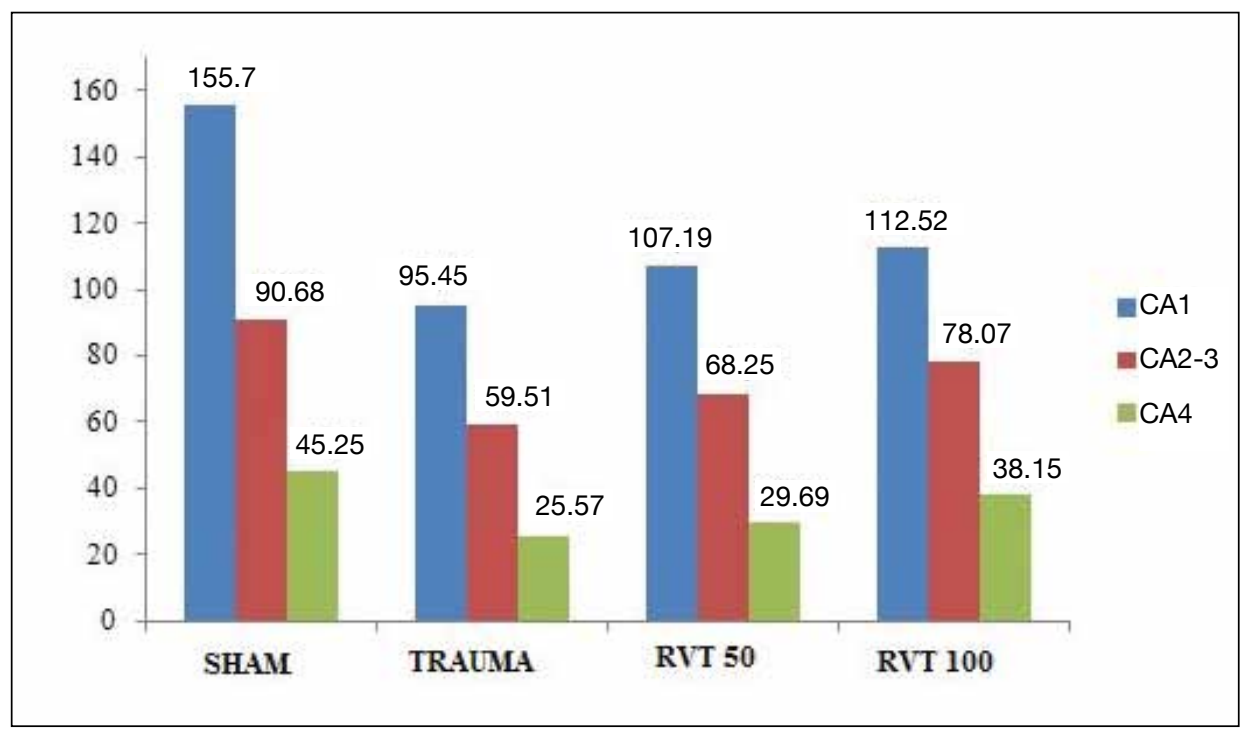

Figure 2: Neuron density among groups.

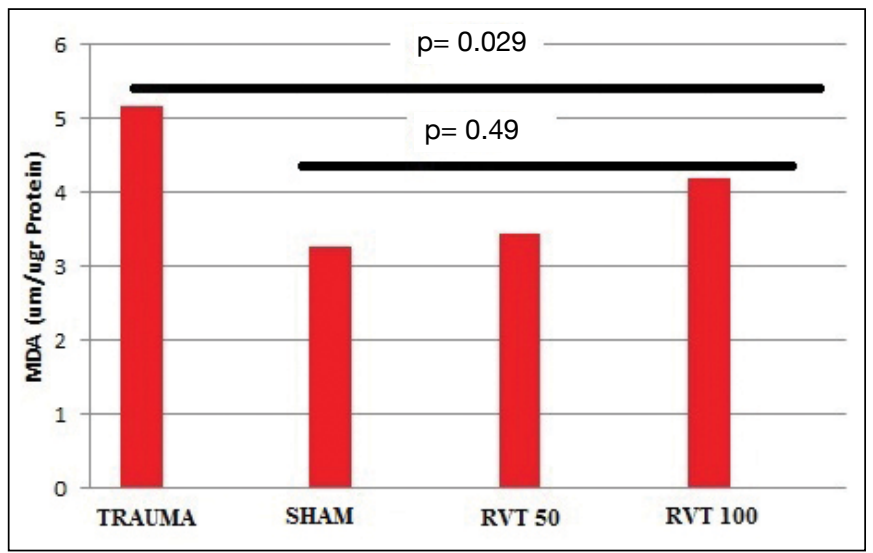

Figure 3: Brain tissue MDA levels among groups.

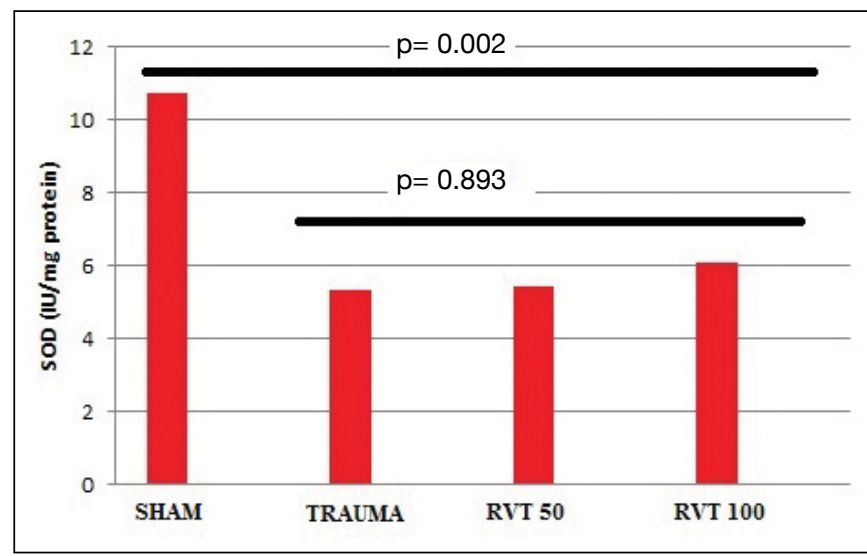

Figure 4: Brain tissue SOD levels among groups. 


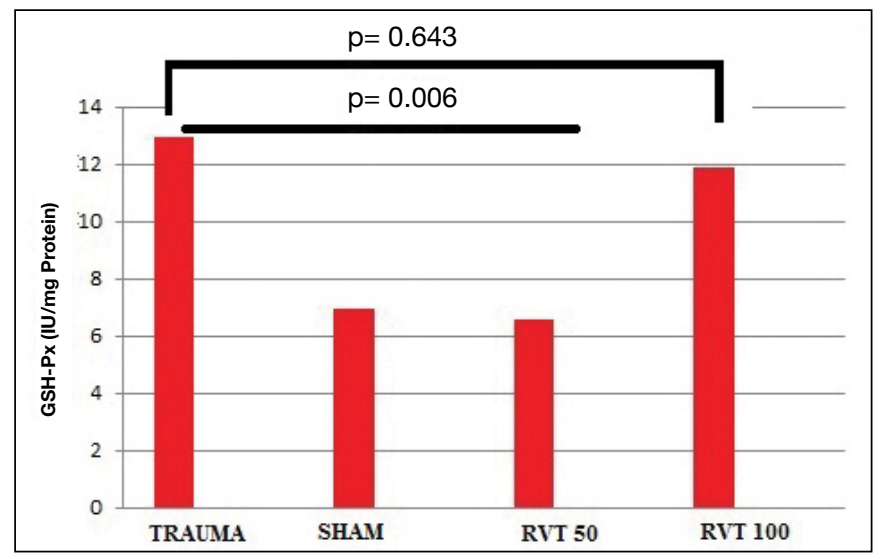

Figure 5: Brain tissue GSH-Px levels among groups.

induced by the primary injury (32). The main course of trauma developed upon injury. We believe that several events followed and increased the neurological impairment through free radical production, parenchymal inflammation, nitric oxide production, lipid peroxidation, and increased intracellular calcium. ROS-mediated lipid peroxidation is an important component of secondary brain injury. Oxygen radicals including hydrogen peroxide, hydroxyl radicals promote secondary brain injury through oxidative stress $(1,11$, $18,21)$. Free radicals are generally limited by cellular oxidative defense mechanisms. Oxidative damage often occurs when free radicals exceed the cellular defense ability and rapidly injure the cell. Antioxidants contribute to mitigating the effects of oxidative stress and include the antioxidant systems SOD, GSH-Px, and CAT $(3,6,8,26,31,34)$.

Neuronal damage in the cortex and hippocampus is caused by direct mechanical impact, cell toxicity, and oxidative mechanisms (33). The death pattern of the neurons after head trauma is a key factor indicating the degree of damage (11). Tang et al. showed that controlled cortical impact causes subcortical neuronal injury as well as widespread white matter damage (38). Özdemir et al. also demonstrated that the cortical impact after head trauma also affects the contralateral hippocampal region. These regions are most prone to damage after TBI (33). Similarly, we demonstrated a significant level of hippocampal cell loss at 7 days after trauma in rats that underwent experimental TBI compared to control animals. Kirino et al. reported that CA1 exhibited increased neuronal cell death relative to the other regions in a transient ischemic model (24). We observed all regions to be sensitive and equally influenced to trauma. We found an increase in neuron counts in all CA1, CA2-3, and CA4 regions in Group 4 than Group 3, which suggest a protective function of RVT. This result was similar to previous reports $(26,31,34)$.

RVT is a polyphenol molecule occurring naturally in wine and grapes, nuts such as pistachios and peanuts, and in bilberries and blueberries. RVT is a potent antioxidant with pharmacological effects on platelet aggregation, lipid metabolism, inflammation, and tumor growth. Experimental evidence suggests that RVT may be a useful therapy in the

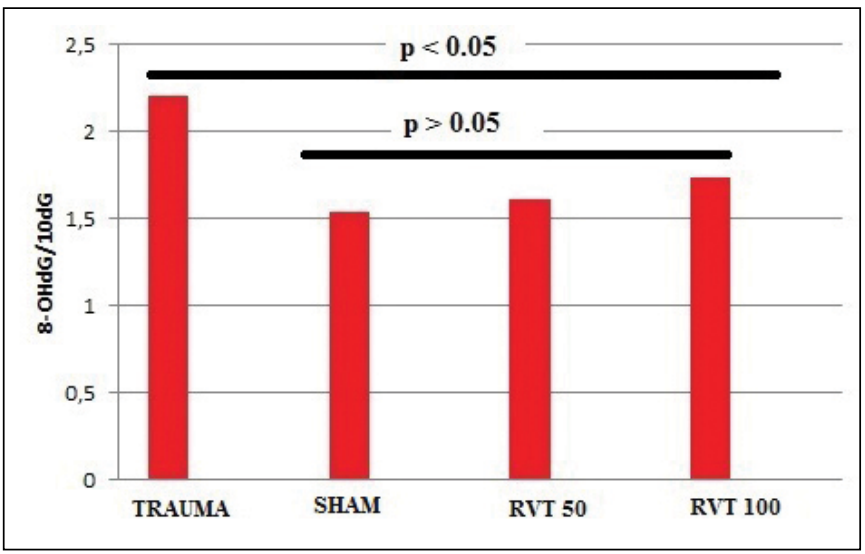

Figure 6: Oxidative DNA damage level among groups.

treatment of cerebral ischemia, epilepsy, and other forms of neuronal cell loss $(7,27,45)$. For example, Liu et al. reported striking functional neurologic protection associated with RVT treatment in experimental models ischemia-reperfusion injury in the spinal cord. The neuroprotectiveness of RVT may promote synergistic benefits in damaged neurons (27).

ROS are primarily generated by the mitochondria, playing an integral part in cerebral ischemic injury (32). ROS is controlled through antioxidants and antioxidases such as GSH, SOD, CAT, and coenzyme Q. SOD is important because it has been used to gauge the ability of the body to remove ROS. On the other hand, MDA levels correlate with the extent of lipid peroxidation. In this study, RVT was shown to enhance SOD and decrease MDA compared to trauma only, suggesting that RVT may protect the mitochondria from oxidative damage.

ROS can elicit DNA base modifications and strand fractures. The most common is the guanine modification, which is altered to $8-\mathrm{OHdG}$ and is a marker of DNA damage (17). We measured 8-OHdG in nucleic acids extracted from brain tissue, relative to dG to determine the proportion of DNA that underwent oxidative damage (17). The levels of $8-\mathrm{OHdG}$ has been shown to increase in patients with degenerative disease and cancer (43). 8-OHdG $/ 10^{6} \mathrm{dG}$ was significantly elevated in rats that underwent $\mathrm{TBI}$ relative to control animals. However, the $8-\mathrm{OHdG} / 10^{6} \mathrm{dG}$ level was lower in the high- and low-dose RVTtreated groups than in the trauma group. The $8-\mathrm{OHdG} / 10^{6} \mathrm{dG}$ level in the RVT-treated groups was comparable to untreated control animals. These results imply that RVT reduces DNA damage. Poly(ADP-ribose) polymerase is involved in DNA repair and can catalyze substitution of $8-O$ OhdG in strands of DNA $(37,40)$. Enzymatic repair of damaged DNA occurs independent of the presence of 8-OHdG, however post-mitotic neurons have low DNA repair capacity and may be particularly vulnerable to damage by modified nucleotides. Dysfunctional DNA maintenance can therefore cause apoptosis in neurons $(37,40,42)$. The observed increase in $8-O H d G-p o s i t i v e$ cells at 1 week after TBI suggests that $8-\mathrm{OHdG}$ accumulations may occur as a result of oxidative damage and dysfunctional DNA repair. 


\section{CONCLUSION}

Resveratrol mitigates damage to neurons following TBI. RVT is associated with increases in the antioxidants GSH-Px and SOD and decreases accumulation of the products of oxidative damage such as MDA and 8-OHdG.

\section{REFERENCES}

1. Aarabi B, Long DM: Dynamics of cerebral edema. The role of an intact vascular bed in the production and propagation of vasogenic brain edema. J Neurosurg 51:779-784, 1979

2. Adeli K, Ogbonna G: Rapid purification of human DNA from whole blood for potential application in clinical chemistry laboratories. Clin Chem 36:261-264, 1990

3. Aebi H: Catalase in vitro. Methods Enzymol 105:121-126, 1984

4. Ak H, Kara M, Gulsen I, Atalay T, Akyol V, Ragbetli MC: Effects of resveratrol on acute sciatic nerve injury in a rat model. $J$ Neurol Sci Turk 32:303-310, 2015

5. Ates O, Cayli S, Altinoz E, Gurses I, Yucel N, Sener M, Kocak A, Yologlu S: Neuroprotection by resveratrol against traumatic brain injury in rats. Mol Cell Biochem 294:137-144, 2007

6. Ates O, Cayli S, Gurses I, Yucel N, Iraz M, Altinoz E, Kocak A, Yologlu S: Effect of pinealectomy and melatonin replacement on morphological and biochemical recovery after traumatic brain injury. Int J Dev Neurosci 24:357-363, 2006

7. Bagriyanik HA, Ersoy N, Cetinkaya C, Ikizoglu E, Kutri D, Ozcana T, Kamanga LG, Kiray M: The effects of resveratrol on chronic constriction injury of sciatic nerve in rats. Neurosci Lett 561:123-127, 2014

8. Bonnefont-Rousselot D, Bastard JP, Jaudon MC, Delattre $\mathrm{J}$ : Consequences of the diabetic status on the oxidant/ antioxidant balance. Diabetes Metab 26:163-176, 2000

9. Cankurt U, Ozbal S, Tekmen I, Erbil G, Sonmez A, Ozogul C: Research into the acute effects of resveratrol on the traumatic brain injury model in immatur rats. J Neurol Sci Turk 30(4): 731-739, 2013

10. Choi SH, Lee SW, Hong YS, Jeun JM, Min BW: Selective inhibition of polymorphonuclear neutrophils by resuscitative concentration of hypertonic saline. Emerg Med J 23:119-122, 2006

11. Demir I, Kiymaz N, Gudu BO, Turkoz Y, Gul M, Dogan Z, Demirtas S: Study of the neuroprotective effect of ginseng on superoxide dismutase (SOD) and glutathione peroxidase (GSH-Px) levels in experimental diffuse head trauma. Acta Neurochir (Wien) 155:913-922, 2013

12. Feeney DM, Boyeson MG, Linn RT, Murray HM, Dail WG: Responses to cortical injury: I. Methodology and local effects of contusions in the rat. Brain Res 211:67-77, 1981

13. Firuzi O, Miri R, Tavakkoli M, Saso L: Antioxidant therapy: current status and future prospects. Curr Med Chem 18:38713888, 2011

14. Frankel EN, Waterhouse AL, Kinsella JE: Inhibition of human LDL oxidation by resveratrol. Lancet 341:1103-1104, 1993
15. Gulsen I, Ak H, Colcimen N, Alp HH, Akyol ME, Demir I, Atalay T, Balahroglu R, Ragbetli MC: Neuroprotective effects of thymoquinone on the hippocampus in a rat model of traumatic brain injury. World Neurosurg 86:243-249, 2016

16. Gundersen H, Bendtsen TF, Korbo L, Marcussen N, Møller A, Nielsen K, Nyengaard J, Pakkenberg B, Sørensen FB, Vesterby A: Some new, simple and efficient stereological methods and their use in pathological research and diagnosis. Apmis 96:379-394, 1988

17. Gutteridge JM, Halliwell B: Antioxidants: Molecules, medicines, and myths. Biochem Biophys Res Commun 393:561564,2010

18. Hall ED: Lipid antioxidants in acute central nervous system injury. Ann Emerg Med 22:1022-1027, 1993

19. Howard V, Reed M: Unbiased stereology: Three-dimensional measurement in microscopy: Garland Science; 2004

20. Jang JH, Surh YJ: Protective effect of resveratrol on betaamyloid-induced oxidative PC12 cell death. Free Radic Biol Med 34:1100-1110, 2003

21. Juurlink B, Paterson P: Review of oxidative stress in brain and spinal cord injury: Suggestions for pharmacological and nutritional management strategies. J Spinal Cord Med 21:309334, 1998

22. Kaur H, Halliwell B: Measurement of oxidized and methylated DNA bases by HPLC with electrochemical detection. Biochem J 318 (Pt 1):21-23, 1996

23. Khoschsorur G, Winklhofer-Roob B, Rabl H, Auer T, Peng Z, Schaur R: Evaluation of a sensitive HPLC method for the determination of malondialdehyde, and application of the method to different biological materials. Chromatographia $52: 181-184,2000$

24. Kirino T, Sano K: Selective vulnerability in the gerbil hippocampus following transient ischemia. Acta Neuropathol 62:201-208, 1984

25. Kunz A, Dirnagl U, Mergenthaler P: Acute pathophysiological processes after ischaemic and traumatic brain injury. Best Pract Res Clin Anaesthesiol 24:495-509, 2010

26. Liao B, Newmark H, Zhou R: Neuroprotective effects of ginseng total saponin and ginsenosides $\mathrm{Rb} 1$ and $\mathrm{Rg} 1$ on spinal cord neurons in vitro. Exp Neurol 173:224-234, 2002

27. Liu C, Shi Z, Fan L, Zhang C, Wang K, Wang B: Resveratrol improves neuron protection and functional recovery in rat model of spinal cord injury. Brain Res 1374:100-109, 2011

28. Maas Al, Stocchetti N, Bullock R: Moderate and severe traumatic brain injury in adults. Lancet Neurol 7:728-741, 2008

29. McIntosh TK, Juhler M, Wieloch T: Novel pharmacologic strategies in the treatment of experimental traumatic brain injury: 1998. J Neurotrauma 15:731-769, 1998

30. Murakami K, Kondo T, Yang G, Chen SF, Morita-Fujimura Y, Chan $\mathrm{PH}$ : Cold injury in mice: A model to study mechanisms of brain edema and neuronal apoptosis. Prog Neurobiol 57:289-299, 1999

31. Naval MV, Gomez-Serranillos MP, Carretero ME, Villar AM: Neuroprotective effect of a ginseng (Panax ginseng) root extract on astrocytes primary culture. J Ethnopharmacol 112:262-270, 2007 
32. Niizuma $\mathrm{K}$, Endo $\mathrm{H}$, Chan $\mathrm{PH}$ : Oxidative stress and mitochondrial dysfunction as determinants of ischemic neuronal death and survival. J Neurochem 109 Suppl 1:133138, 2009

33. Ozdemir D, Tugyan K, Uysal N, Sonmez U, Sonmez A, Acikgoz O, Ozdemir N, Duman M, Ozkan H: Protective effect of melatonin against head trauma-induced hippocampal damage and spatial memory deficits in immature rats. Neurosci Lett 385:234-239, 2005

34. Rausch WD, Liu S, Gille G, Radad K: Neuroprotective effects of ginsenosides. Acta Neurobiol Exp (Wars) 66:369-375, 2006

35. Reilly PL: Brain injury: The pathophysiology of the first hours.' Talk and Die revisited'. J Clin Neurosci 8:398-403, 2001

36. Sun Y, Oberley LW, Li Y: A simple method for clinical assay of superoxide dismutase. Clin Chem 34:497-500, 1988

37. Takizawa Y, Miyazawa T, Nonoyama S, Goto Y, Itoh M: Edaravone inhibits DNA peroxidation and neuronal cell death in neonatal hypoxic-ischemic encephalopathy model rat. Pediatr Res 65:636-641, 2009

38. Tang Q, Li G, Wei X, Zhang J, Chiu JF, Hasenmayer D, Zhang $\mathrm{D}$, Zhang $\mathrm{H}$ : Resveratrol-induced apoptosis is enhanced by inhibition of autophagy in esophageal squamous cell carcinoma. Cancer Lett 336:325-337, 2013
39. Tarng DC, Huang TP, Wei YH, Liu TY, Chen HW, Wen Chen T, Yang WC: 8-hydroxy-2'-deoxyguanosine of leukocyte DNA as a marker of oxidative stress in chronic hemodialysis patients. Am J Kidney Dis 36:934-944, 2000

40. Wang X, Karlsson JO, Zhu C, Bahr BA, Hagberg H, Blomgren $\mathrm{K}$ : Caspase-3 activation after neonatal rat cerebral hypoxiaischemia. Biol Neonate 79:172-179, 2001

41. Watts DD, Hanfling D, Waller MA, Gilmore C, Fakhry SM, Trask AL: An evaluation of the use of guidelines in prehospital management of brain injury. Prehosp Emerg Care 8:254-261, 2004

42. Weissman L, de Souza-Pinto NC, Stevnsner T, Bohr VA: DNA repair, mitochondria, and neurodegeneration. Neuroscience 145:1318-1329, 2007

43. Yao X, Zhong L: Genotoxic risk and oxidative DNA damage in HepG2 cells exposed to perfluorooctanoic acid. Mutat Res 587:38-44, 2005

44. Zamin LL, Dillenburg-Pilla P, Argenta-Comiran R, Horn AP, Simão F, Nassif M, Gerhardt D, Frozza RL, Salbego C: Protective effect of resveratrol against oxygen-glucose deprivation in organotypic hippocampal slice cultures: Involvement of PI3-K pathway. Neurobiol Dis 24:170-182, 2006

45. Zhang F, Liu J, Shi JS: Anti-inflammatory activities of resveratrol in the brain: Role of resveratrol in microglial activation. Eur $\mathrm{J}$ Pharmacol 636:1-7, 2010 